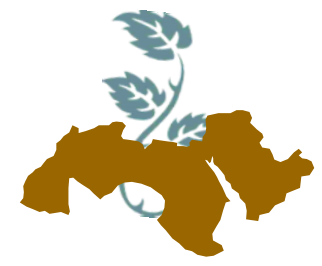

\title{
A STUDY ON THE WEEDS DIVERSITY ALONG MARIOTTEYA CANAL AT GIZA GOVERNORATE, EGYPT
}

\author{
Safwat A. Azer \\ Flora and Phytotaxonomy Researches Dept., Horticultural Research Institute, \\ Agricultural Research Center, Giza, Egypt \\ Corresponding author: E-mail: safwat.azer@hotmail.com
}

Keywords: Weed diversity, Life cycle, Life form, Chorology, Mariotteya Canal

\begin{abstract}
This research aims to study weeds diversity among five represented habitats namely; field crops, orchards, canal banks, wastelands and water bodies along Mariotteya Canal, Giza. A total of 88 species belong to 74 genera and 27 families were recorded and identified with Egyptian floras and available checklists. The dicotyledons families were represented by (69\%); while monocotyledons were $(31 \%)$. The common families were Poaceae $(26 \%)$; while both of Compositae and Leguminosae were represented by $(8 \%)$. Three species were recorded from each of Apiaceae, Cyperaceae, Malvaceae and Polygonaceae and two species from Salicaceae and Amaranthaceae. In addition to, 13 families were monotypic. The annuals species were represented by (59\%), perennial herbs were $(30 \%)$; while trees, shrubs and biennials species were $(7 \%),(3 \%)$ and $(1 \%)$; respectively. The life form showed that, therophytes species were represented by $(60 \%)$ followed by chamaephytes $(14 \%)$ then phanerophytes (10\%). The chorological ratios showed that cosmopolitan taxa had the highest contribution (25\%), followed by pantropical $(19 \%)$ then palaeotropical (14\%). Based on species recorded among five represented habitats, the cluster analysis divided the studied habitats into three groups. Group one included field crops and orchards habitats. Second group contained canal banks and wastelands habitats; while third group included water bodies' habitat. Moreover, field crops and orchards habitats showed the highest similarity value (0.614) followed by $(0.559)$ between canal banks and wastelands habitats. On
\end{abstract}

the other hand, there was no similarity between species of water bodies' habitat and species of the other habitats.

\section{INTRODUCTION}

The number of weeds recorded in the flora of Egypt increased to 470 species which constitute the current weed assemblages of Egypt and represent about $22.5 \%$ of the total number of the flowering plants in the Flora of Egypt (Boulos 1995). Many studies were conducted on the weed flora of the Nile islands and irrigation canals; El Hadidi et al (1996) gave accounts with different aspects of diversity in the weed flora among different phytogeographical regions of Egypt. Mohamed and Hassan (1998) conducted a study on the plant life in the Nile islands of Minia governorate and recorded about 95 species. El Hadidi and Hosni, (2000) studied weeds diversity in the flora of Egypt. Shaheen (2002) gave an account on some aspects of biodiversity of the weed flora in the farmland of different phytogeographical regions of Aswan. Also, Shaheen et al (2004) recorded 206 species in a study of botanical diversity in the flora of some islands in the Egyptian Nubia. Mashaly and El-Ameir (2007) recorded 70 species through study on hydrophytic vegetation in the irrigation and drainage canal system of the River Nile in the Delta region.

Irrigation canals transport water from the water source to the farmer's fields. The more fields that are served by a canal, the more water have to be transported. River Nile is the main fresh water resource in Egypt, meeting all demands for drinking water, irrigation and industry. In Egypt, the total length of canals and drains is approximately 4700 km (Bliek Van der et al 1982). These canals and 
drains are infested by different aquatic weeds. The degree of infestation is affected by environmental factors, including water transparency, depth of water, water currents and air temperature (EIGharably et al 1982). Mariotteya canal is used for irrigation and drainage and runs directly from south border of Giza governorate passing through Cairo, Nile Delta till King Marriott at Alexandria governorate.

The different life forms of aquatic vegetation were defined in different parts of Egypt by many authors, among of them: Springuel \& Murphy (1991); Ali et al (1995); Shaltout et al (1995); Serag \& Khedr (1996); Khedr \& El-Demerdash (1997); Khedr (1998); Serag, et al (1999); Mashaly et al (2003) and Zahran \& Willis (2003). This study is concerning with the part of Mariotteya Canal through Giza governorate which extends from Al-Ayat till Abu Rawash areas for $80 \mathrm{~km}$ long. The aim of this study is to provide a description of weeds diversity along Mariotteya canal and demonstrate life cycle and life form ratios and the chorological affinities of the collected species among five represented habitats namely; field crops, orchards, canal banks, wastelands and water bodies.

\section{MATERIALS AND METHODS}

\section{The Study Area}

The studied area from mariotteya canal belongs to Giza governorate and extends about 80 km from Al-Ayat till Abu Rawash areas and located between latitude 2958'30"n and longitude $31^{\circ} 10^{\prime} 15^{\prime \prime}$ e (Figure 1). The studied area was represented by five different habitats namely; field crops, orchards, canal banks, wastelands and water bodies. Based on the climatic data, the mean annual temperature and rainfall are $25.25^{\circ} \mathrm{C}$ and $1.15 \mathrm{~mm}$; respectively. Most of the rains fall in winter months.

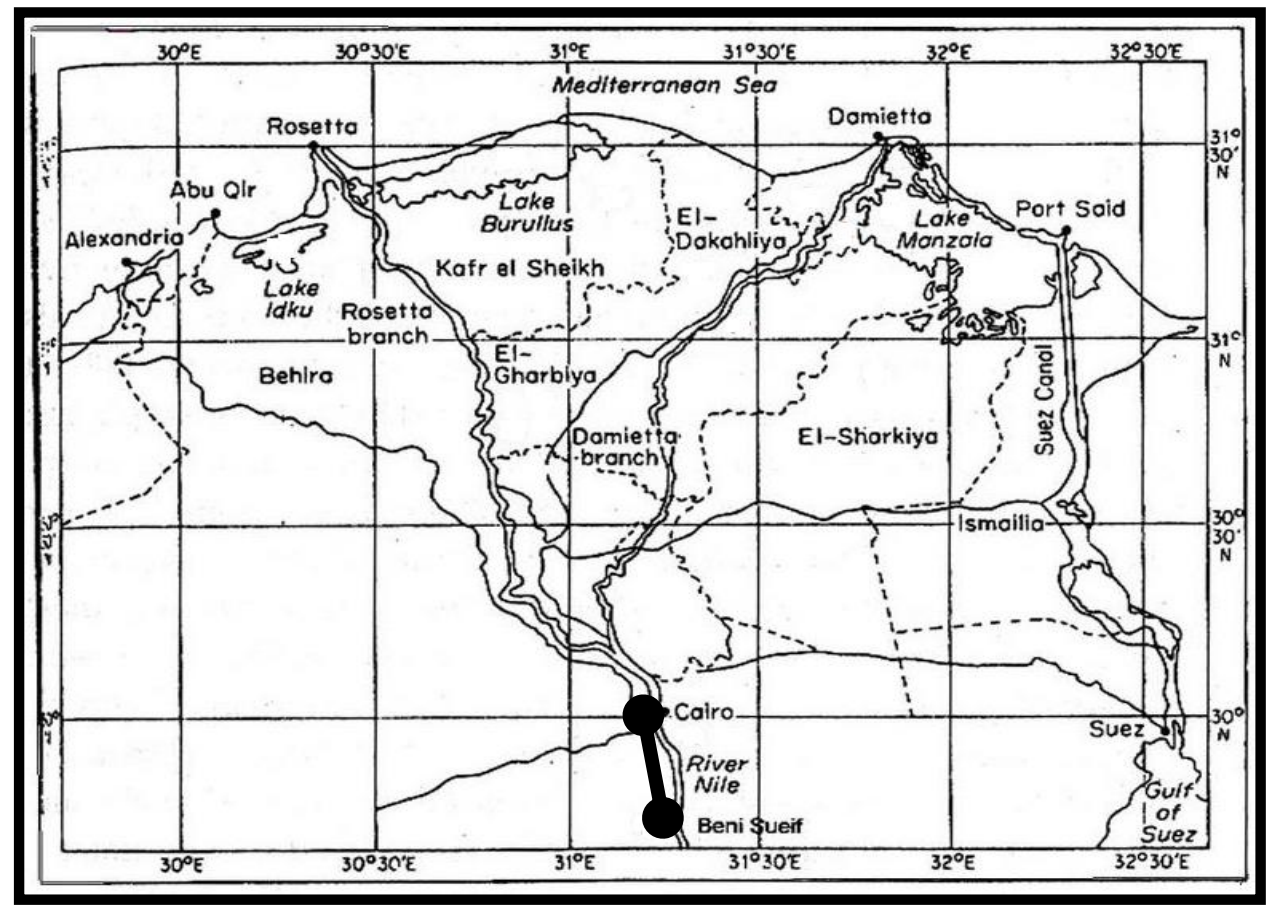

Fig. 1. A map showing the studied area along Mariotteya Canal at Giza governorate

\section{Data collection}

Several visits were carried out along the studied area of Mariotteya Canal to cover all the represented habitats from February 2016 till February 2017. Field data of the weeds diversity, among five represented habitats were gathered. The studied species were recorded to represent the weeds diversity and covered the represented habitats along Mariotteya canal. The recorded species were arranged alphabetically with their families (Table 1). 


\section{Species identification}

Species identification was based on Täckholm (1974); Boulos (1995, 1999, 2000, 2002, 2005 \& 2009) and updated by Angiosperm Phylogeny Group III (2009); Chase and Reveal (2009) and Haston et al (2009). Life forms (Therophytes, Geophytes, Chamaephytes,Hemicryptophytes, Phanerophytes, helophytes and hydrophytes) were identified according to Raunkiaer's system of classification (Raunkiaer 1934). Chorology (Cosmopolitan, Pantropical, Palaeotropical, Mediterranean, Irano- Turanian, Sudano-Zambezian, EuroSiberian and Saharo-Sindian) were cited according to Zohary (1966 \& 1987); Wickens (1976) and Feinbrun-Dothan (1978 \& 1985). Voucher specimens were collected and identified at the herbarium of Flora and Phytotaxonomy Research Department (CAIM), Horticultural Research Institute, Agricultural Research Center, Giza, Egypt and arranged alphabetically with their families. About 240 plant specimens were collected and prepared as herbarium sheets.

\section{Numerical analysis}

Numerical analysis of the five represented habitats was carried out and based on hierarchical cluster analysis. The retrieved output was used to construct specific ecological relationships among the collected species. The substantial numbers ( $1=$ presence and $0=$ absence) of the recorded species were used for each selected habitat (Table 1). The data treated as a Pearson correlation in a data matrix to measure degree of similarity value using SPSS version 22 (SPSS, 2013). The output was plotted in the form of UPGMA (Unweighted pair group method with arithmetic mean) dendrogram (Figure 8). The dendrogram was based on average linkage (between groups) and rescaled distance cluster combine.

\section{RESULTS}

\section{Floristic analysis}

The study recorded the presence of 88 species, representing 74 genera and 27 families. Dicotyledons species were represented by $(69 \%)$; while monocotyledons species were (31\%) (Figure 2). The most distributed families were Poaceae followed by Compositae and Leguminosae then Brassicaceae. Poaceae were represented by (26\%). Both of Compositae and Leguminosae were $(8 \%)$; while Brassicaceae was $(7 \%)$. Moreover, each of Chenopodiaceae, Convolvulaceae, Euphorbiaceae and Solanaceae was (5\%). In addition to, three species were recorded from each of Apiaceae, Cyperaceae, Malvaceae and Polygonaceae and two species from Amaranthaceae and Salicaceae; while 13 families were monotypic (Figure 3). The recorded species were arranged alphabetically with their families and genera and the presence or absence values in the studied habitats are listed in (Table 1).

\section{Life cycle ratios}

The life cycle ratios of the studied species outlined in (Figure 4) revealed the annuals species dominated the flora of Mariotteya Canal. These represented by $(59 \%)$ followed by perennial herbs $(30 \%)$. Moreover, trees and shrubs were represented by $(7 \%)$ and $(3 \%)$; respectively while biennials species were $(1 \%)$.

\section{Life form ratios}

The life forms of the collected taxa revealed that the therophytes species dominated the flora of Mariotteya Canal. These represented by $(60 \%)$, followed by chamaephytes (14\%) then phanerophytes (10\%). In addition to, both of hemicryptophytes and hydrophytes were represented by $(5 \%)$; while both of geophytes and helophytes were (3\%) (Figure 5).

\section{Chorological ratios}

It was obvious that cosmopolitan have the highest ratio $(25 \%)$ followed by pantropical (19\%) and palaeotropical (14\%). The mono-regional species dominated by Mediterranean, SudanoZambezian, Saharo-Sindian and Sudanian regions were represented by $(6 \%),(3 \%),(2 \%)$ and $(1 \%)$; respectively. Moreover, the bi-regional species dominated by (Mediterranean \& Irano-Turanian) regions constituted (9\%) followed by (SaharoSindian \& Sudano-Zambezian) regions (7\%), while both of (Irano-Turanian \& Saharo-Sindian) and (Mediterranean \& Saharo-Sindian) regions were (1\%). In addition to, the tri-regional species dominated by (Mediterranean, Irano-Turanian \& Saharo-Sindian) regions constituted $(5 \%)$ followed by (Mediterranean, Irano-Turanian \& Euro-Siberian) regions (4\%); while (Mediterranean, Euro-Siberian \& Saharo-Sindian) and (Irano-Turanian, SaharoSindian \& Sudano- Zambezian) were $(2 \%)$ and 
Table 1. The species recorded in the studied habitats within their families, life cycle, life form, chorology and their presence or absence along Mariotteya Canal.

\begin{tabular}{|c|c|c|c|c|c|c|c|c|c|}
\hline \multirow[b]{2}{*}{ No } & \multirow[b]{2}{*}{ Taxa } & \multirow[b]{2}{*}{ 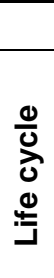 } & \multirow[b]{2}{*}{ 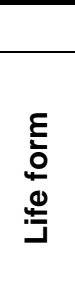 } & \multirow[b]{2}{*}{$\begin{array}{l}\text { 응 } \\
\frac{0}{0} \\
\frac{0}{0} \\
\frac{}{0}\end{array}$} & \multicolumn{5}{|c|}{ Habitat types } \\
\hline & & & & & $\begin{array}{l}\text { 음 } \\
\frac{0}{0} \\
\frac{0}{0} \\
\frac{0}{4}\end{array}$ & $\begin{array}{l}\frac{n}{0} \\
\frac{0}{\pi} \\
\frac{0}{0} \\
\text { o }\end{array}$ & 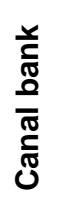 & $\begin{array}{l}\frac{n}{0} \\
\frac{0}{c} \\
\frac{0}{0} \\
\frac{d}{0} \\
\stackrel{0}{0} \\
3\end{array}$ & 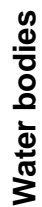 \\
\hline 1 & Amaranthaceae & & & & & & & & \\
\hline 1.1 & Amaranthus lividus $\mathrm{L}$. & $A$ & Th & Cosm & 1 & 1 & 0 & 1 & 0 \\
\hline 1.2 & Amaranthus viridis $\mathrm{L}$. & $A$ & Th & Pal & 1 & 1 & 0 & 0 & 0 \\
\hline 2 & Apiaceae & & & & & & & & \\
\hline 2.3 & Ammi majus $\mathrm{L}$. & $A$ & Th & SZ & 1 & 0 & 0 & 0 & 0 \\
\hline 2.4 & Ammi visnaga (L.) Lam. & $A$ & Th & $M$ & 1 & 0 & 0 & 0 & 0 \\
\hline 3.5 & $\begin{array}{l}\text { Cyclospermum leptophyllum (Pers.) } \\
\text { Sprague }\end{array}$ & A & Th & Pal & 1 & 0 & 0 & 0 & 0 \\
\hline 3 & Apocynaceae & & & & & & & & \\
\hline 4.6 & Cynanchum acutum L. & $\mathrm{P}$ & $\mathrm{Hm}$ & M,IT,SS & 0 & 1 & 1 & 1 & 0 \\
\hline 4 & Araceae & & & & & & & & \\
\hline 5.7 & Lemna gibba L. & $\mathrm{P}$ & Hy & M,IT,SS & 0 & 0 & 0 & 0 & 1 \\
\hline 5 & Arecaceae & & & & & & & & \\
\hline 6.8 & Phoenix dactylifera L. & $\mathrm{T}$ & $\mathrm{Ph}$ & $\mathrm{M}, \mathrm{IT}$ & 1 & 1 & 1 & 1 & 0 \\
\hline 6 & Brassicaceae & & & & & & & & \\
\hline 7.9 & Brassica tournefortii Gouan & $A$ & Th & $\mathrm{M}, \mathrm{IT}$ & 1 & 1 & 0 & 0 & 0 \\
\hline 8.10 & Eruca vesicaria (L.) Cav. & $A$ & Th & $\mathrm{M}$ & 1 & 1 & 1 & 0 & 0 \\
\hline 9.11 & Lepidium sativum $\mathrm{L}$. & $A$ & Th & Cosm & 1 & 0 & 0 & 0 & 0 \\
\hline 10.12 & Raphanus raphanistrum L. & $A$ & Th & Cosm & 1 & 1 & 0 & 0 & 0 \\
\hline 11.13 & Sinapis alba L. & $A$ & Th & Pan & 1 & 1 & 0 & 0 & 0 \\
\hline 12.14 & Sisymbrium irio L. & $A$ & Th & M,IT,ES & 0 & 1 & 1 & 0 & 0 \\
\hline 7 & Ceratophyllaceae & & & & & & & & \\
\hline 13.15 & Ceratophyllum demersum L. & $\mathrm{P}$ & Hy & Cosm & 0 & 0 & 0 & 0 & 1 \\
\hline 8 & Chenopodiaceae & & & & & & & & \\
\hline 14.16 & Bassia indica (Wight) A.J.Scott & $A$ & Th & IT,SS & 0 & 0 & 1 & 0 & 0 \\
\hline 15.17 & Beta vulgaris $\mathrm{L}$. & $A$ & Th & SS & 1 & 1 & 0 & 0 & 0 \\
\hline 16.18 & Chenopodium album L. & $A$ & Th & Cosm & 1 & 1 & 1 & 1 & 0 \\
\hline 16.19 & Chenopodium murale L. & $A$ & Th & Cosm & 1 & 1 & 1 & 1 & 0 \\
\hline 9 & Compositae & & & & & & & & \\
\hline 17.20 & Bidens pilose $\mathrm{L}$. & $A$ & Th & Pan & 0 & 0 & 1 & 1 & 0 \\
\hline 18.21 & $\begin{array}{l}\text { Cichorium endivia L. subsp. divarica- } \\
\text { tum (Schousb.) P.D. Sell }\end{array}$ & $A$ & Th & $\mathrm{M}, \mathrm{IT}$ & 1 & 1 & 1 & 0 & 0 \\
\hline 19.22 & Eclipta prostrata (L.) L. & $A$ & Th & Pan & 0 & 0 & 1 & 1 & 0 \\
\hline 20.23 & Erigeron bonariensis $\mathrm{L}$. & $A$ & Th & $\mathrm{M}$ & 0 & 1 & 1 & 1 & 0 \\
\hline 21.24 & Lactuca serriola L. & $\mathrm{B}$ & Th & Cosm & 0 & 0 & 1 & 1 & 0 \\
\hline 22.25 & Pluchea dioscoridis (L.) DC. & $S$ & $\mathrm{Ch}$ & Cosm & 0 & 0 & 1 & 0 & 0 \\
\hline 23.26 & Sonchus oleraceus (L.) L. & A & Th & Cosm & 1 & 1 & 1 & 0 & 0 \\
\hline
\end{tabular}


Table 1. Continued

\begin{tabular}{|c|c|c|c|c|c|c|c|c|c|}
\hline \multirow[b]{2}{*}{ No } & \multirow[b]{2}{*}{ Taxa } & \multirow[b]{2}{*}{ 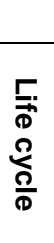 } & \multirow[b]{2}{*}{$\begin{array}{l}\overline{\bar{\sigma}} \\
\overrightarrow{0} \\
\frac{\overrightarrow{0}}{3}\end{array}$} & \multirow[b]{2}{*}{$\begin{array}{l}\text { 웅 } \\
\frac{0}{0} \\
\frac{0}{0} \\
\stackrel{0}{<}\end{array}$} & \multicolumn{5}{|c|}{ Habitat types } \\
\hline & & & & & 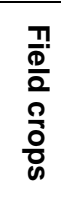 & $\begin{array}{l}\text { 음 } \\
\frac{1}{2} \\
\frac{2}{2} \\
\frac{0}{\infty}\end{array}$ & 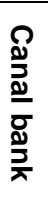 & 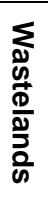 & 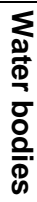 \\
\hline 10 & Convolvulaceae & & & & & & & & \\
\hline 24.27 & Convolvulus arvensis L. & $P$ & $\mathrm{Ch}$ & Pal & 1 & 1 & 1 & 1 & 0 \\
\hline 25.28 & Cuscuta campestris Yunck. & A & Th & Pan & 1 & 0 & 0 & 0 & 0 \\
\hline 26.29 & Ipomoea carnea Jacq. & S & $\mathrm{Ph}$ & Pan & 0 & 0 & 1 & 1 & 0 \\
\hline 26.30 & Ipomoea cairica (L.) Sweet & $P$ & $\mathrm{Ch}$ & Pan & 0 & 0 & 1 & 1 & 0 \\
\hline 11 & Cyperaceae & & & & & & & & \\
\hline 27.31 & Cyperus alopecuroides Rottb. & $P$ & $\mathrm{Ch}$ & Pan & 0 & 0 & 1 & 0 & 0 \\
\hline 27.32 & Cyperus articulatus L. & $P$ & $\mathrm{Ch}$ & Pal & 0 & 0 & 1 & 0 & 0 \\
\hline 27.33 & Cyperus rotundus L. & $P$ & $\mathrm{Ge}$ & Pan & 1 & 1 & 1 & 1 & 0 \\
\hline 12 & Euphorbiaceae & & & & & & & & \\
\hline 28.34 & Euphorbia heterophylla L. & A & Th & Cosm & 0 & 1 & 1 & 1 & 0 \\
\hline 28.35 & Euphorbia peplus L. & A & Th & Pan & 1 & 1 & 1 & 0 & 0 \\
\hline 28.36 & Euphorbia prostrata Aiton & $A$ & Th & Cosm & 0 & 0 & 1 & 1 & 0 \\
\hline 29.37 & Ricinus communis $\mathrm{L}$. & $S$ & $\mathrm{Ph}$ & M & 0 & 0 & 1 & 1 & 0 \\
\hline 13 & Lamiaceae & & & & & & & & \\
\hline 30.38 & $\begin{array}{l}\text { Mentha longifolia subsp. typhoides (Briq.) } \\
\text { Harley }\end{array}$ & $\mathrm{P}$ & Ch & Pal & 0 & 0 & 1 & 0 & 0 \\
\hline 14 & Leguminosae & & & & & & & & \\
\hline 31.39 & Acacia nilotica (L.) Willd. ex Del. & $\mathrm{T}$ & $\mathrm{Ph}$ & $\mathbf{S}$ & 0 & 0 & 1 & 0 & 0 \\
\hline 32.40 & Alhagi graecorum Boiss. & $\mathrm{P}$ & $\mathrm{Hm}$ & Pal & 0 & 1 & 1 & 1 & 0 \\
\hline 33.41 & Leucaena leucocephala (Lam.) De Wit & $\mathrm{T}$ & $\mathrm{Ph}$ & Pal & 0 & 0 & 1 & 0 & 0 \\
\hline 34.42 & Melilotus indicus (L.) All. & $A$ & Th & M,ES,SS & 1 & 1 & 0 & 0 & 0 \\
\hline 35.43 & Sesbania sesban (L.) Merr. & $\mathrm{S}$ & $\mathrm{Ph}$ & M,IT & 0 & 0 & 1 & 1 & 0 \\
\hline 36.44 & Trifolium alexandrinum $\mathrm{L}$. & A & Th & M & 1 & 1 & 1 & 0 & 0 \\
\hline 36.45 & Trifolium resupinatum L. & $A$ & Th & Pal & 1 & 1 & 1 & 0 & 0 \\
\hline 15 & Malvaceae & & & & & & & & \\
\hline 37.46 & Corchorus olitorius L. & $A$ & Th & Pan & 1 & 1 & 1 & 0 & 0 \\
\hline 38.47 & Malva parviflora L. & $A$ & Th & M,IT & 1 & 1 & 1 & 1 & 0 \\
\hline 39.48 & Sida spinosa L. & $\mathrm{P}$ & $\mathrm{Ch}$ & Cosm & 0 & 1 & 1 & 1 & 0 \\
\hline 16 & Plantaginaceae & & & & & & & & \\
\hline 40.49 & Plantago major L. & $A$ & Th & $\mathrm{M}, \mathrm{IT}$ & 1 & 1 & 0 & 0 & 0 \\
\hline 17 & Poaceae & & & & & & & & \\
\hline 41.50 & Avena fatua $\mathrm{L}$. & $A$ & Th & Cosm & 1 & 0 & 0 & 0 & 0 \\
\hline 41.51 & Avena sterilis $\mathrm{L}$. & $A$ & Th & $\mathrm{M}, \mathrm{IT}$ & 1 & 0 & 0 & 0 & 0 \\
\hline 42.52 & Brachiaria mutica (Forssk.) Stapf & $A$ & Th & Cosm & 1 & 1 & 0 & 1 & 0 \\
\hline 43.53 & Cenchrus barbatus Schum. & & & & 0 & 1 & 1 & 1 & 0 \\
\hline 43.54 & Cenchrus biflorus Roxb. & $A$ & Th & SS,SZ & 0 & 1 & 1 & 1 & 0 \\
\hline 44.55 & Cynodon dactylon (L.) Pers. & $\mathrm{P}$ & $\mathrm{Ge}$ & IT,SS,SZ & 1 & 1 & 1 & 1 & 0 \\
\hline 45.56 & Desmostachya bipinnata (L.) Stapf & $P$ & $\mathrm{Ge}$ & SS,SZ & 0 & 0 & 1 & 0 & 0 \\
\hline 46.57 & Digitaria sanguinalis (L.) Scop. & $A$ & Th & Pal & 1 & 1 & 1 & 0 & 0 \\
\hline 47.58 & Echinochloa colona (L.) Link & A & Th & Pan & 1 & 1 & 1 & 1 & 0 \\
\hline 47.59 & Echinochloa stagnina (Retz.) P. Beauv. & $\mathrm{P}$ & $\mathrm{Ge}$ & Pal & 1 & 1 & 1 & 1 & 0 \\
\hline 48.60 & Imperata cylindrica (L.) Raeusch. & $P$ & $\mathrm{Hm}$ & SS,SZ & 0 & 0 & 1 & 1 & 0 \\
\hline 49.61 & Leptochloa fusca (L.) Kunth & $\mathrm{P}$ & $\mathrm{Ch}$ & Pal & 0 & 1 & 1 & 1 & 0 \\
\hline 50.62 & Lolium multiflorum Lam. & A & Th & Pan & 1 & 1 & 1 & 0 & 0 \\
\hline
\end{tabular}


Table 1. Continued

\begin{tabular}{|c|c|c|c|c|c|c|c|c|c|}
\hline \multirow[b]{2}{*}{ No } & \multirow[b]{2}{*}{ Taxa } & \multirow[b]{2}{*}{$\begin{array}{l}\frac{0}{0} \\
\text { ত̃ } \\
\stackrel{0}{\Xi}\end{array}$} & \multirow[b]{2}{*}{ 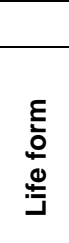 } & \multirow[b]{2}{*}{$\begin{array}{l}\text { 흐 } \\
\text { 음 } \\
\text { 흠 }\end{array}$} & \multicolumn{5}{|c|}{ Habitat types } \\
\hline & & & & & $\begin{array}{l}\text { : } \\
\frac{0}{0} \\
\frac{0}{0} \\
\frac{0}{0} \\
\frac{0}{4}\end{array}$ & 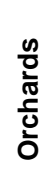 & 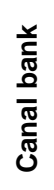 & $\begin{array}{l}\frac{0}{0} \\
\frac{c}{\pi} \\
\frac{\pi}{0} \\
\frac{\pi}{3} \\
\frac{\pi}{3}\end{array}$ & 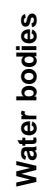 \\
\hline 51.63 & Panicum coloratum L. & & & & 1 & 1 & 0 & 0 & 0 \\
\hline 52.64 & $\begin{array}{l}\text { Paspalidium geminatum (Forssk.) } \\
\text { Stapf }\end{array}$ & $\mathrm{P}$ & $\mathrm{Ch}$ & Cosm & 0 & 1 & 1 & 1 & 0 \\
\hline 53.65 & Phalaris minor Retz. & A & Th & Pan & 0 & 1 & 1 & 0 & 0 \\
\hline 54.66 & $\begin{array}{l}\text { Phragmites australis (Cav.) Trin. } \\
\text { ex Steud. }\end{array}$ & $P$ & $\mathrm{He}$ & SS,SZ & 0 & 0 & 1 & 1 & 0 \\
\hline 55.67 & Poa annua L. & A & Th & M,IT,SS & 0 & 1 & 1 & 0 & 0 \\
\hline 56.68 & $\begin{array}{l}\text { Polypogon monspeliensis (L.) } \\
\text { Desf. }\end{array}$ & A & Th & M,IT,ES & 1 & 1 & 1 & 1 & 0 \\
\hline 57.69 & Saccharum spontaneum L. & $P$ & $\mathrm{Hm}$ & M,SS & 0 & 0 & 1 & 0 & 0 \\
\hline 58.70 & Setaria verticillata (L.) P. Beauv. & A & Th & SZ & 1 & 1 & 1 & 1 & 0 \\
\hline 58.71 & Setaria viridis (L.) P. Beauv. & A & Th & Cosm & 1 & 1 & 1 & 1 & 0 \\
\hline $\begin{array}{l}59.72 \\
18\end{array}$ & $\begin{array}{l}\text { Sorghum virgatum (Hack.) Stapf } \\
\text { Polygonaceae }\end{array}$ & B & Th & Cosm & 0 & 0 & 1 & 0 & 0 \\
\hline 60.73 & Emex spinosa (L.) Campd. & A & Th & M,IT,SS & 1 & 1 & 1 & 0 & 0 \\
\hline 61.74 & $\begin{array}{l}\text { Persicaria senegalensis (Meisn.) } \\
\text { Soják }\end{array}$ & $P$ & $\mathrm{He}$ & SS & 0 & 0 & 0 & 0 & 1 \\
\hline $\begin{array}{l}62.75 \\
19\end{array}$ & $\begin{array}{l}\text { Rumex dentatus } \mathrm{L} . \\
\text { Pontederiaceae }\end{array}$ & $A$ & Th & Cosm & 1 & 1 & 1 & 0 & 0 \\
\hline $\begin{array}{l}63.76 \\
20\end{array}$ & $\begin{array}{l}\text { Eichhornia crassipes (Mart.) Solms } \\
\text { Portulacaceae }\end{array}$ & $P$ & $\mathrm{Hy}$ & Pal & 0 & 0 & 0 & 0 & 1 \\
\hline $\begin{array}{l}64.77 \\
21\end{array}$ & $\begin{array}{l}\text { Portulaca oleracea } \mathrm{L} \text {. } \\
\text { Potamogetonaceae }\end{array}$ & $A$ & Th & Cosm & 1 & 1 & 1 & 1 & 0 \\
\hline $\begin{array}{l}65.78 \\
22\end{array}$ & $\begin{array}{l}\text { Potamogeton nodosus Poir. } \\
\text { Primulaceae }\end{array}$ & $P$ & $\mathrm{Hy}$ & Cosm & 0 & 0 & 0 & 0 & 1 \\
\hline $\begin{array}{l}66.79 \\
23\end{array}$ & $\begin{array}{l}\text { Anagallis arvensis } \mathrm{L} . \\
\text { Salicaceae }\end{array}$ & $A$ & Th & Cosm & 1 & 0 & 1 & 0 & 0 \\
\hline 67.80 & Salix mucronata Thunb. & $\mathrm{T}$ & $\mathrm{Ph}$ & Pan & 0 & 0 & 1 & 1 & 0 \\
\hline $\begin{array}{l}67.81 \\
24\end{array}$ & $\begin{array}{l}\text { Salix tetrasperma Roxb. } \\
\text { Solanaceae }\end{array}$ & $\mathrm{T}$ & $\mathrm{Ph}$ & $\mathrm{Pal}$ & 0 & 0 & 1 & 1 & 0 \\
\hline 68.82 & Datura stramonium $\mathrm{L}$. & A & Th & Pan & 0 & 0 & 1 & 1 & 0 \\
\hline 69.83 & Lycopersicon esculentum Mill. & A & Th & SZ & 1 & 1 & 1 & 0 & 0 \\
\hline 70.84 & Solanum americanum Mill. & A & Th & Cosm & 0 & 1 & 1 & 1 & 0 \\
\hline $\begin{array}{l}71.85 \\
25\end{array}$ & $\begin{array}{l}\text { Withania somnifera (L.) Dunal } \\
\text { Tamaricaceae }\end{array}$ & $\mathrm{P}$ & $\mathrm{Ch}$ & SS,SZ & 0 & 0 & 1 & 1 & 0 \\
\hline $\begin{array}{l}72.86 \\
26\end{array}$ & $\begin{array}{l}\text { Tamarix senegalensis DC. } \\
\text { Typhaceae }\end{array}$ & $\mathrm{T}$ & $\mathrm{Ph}$ & M,ES,SS & 0 & 0 & 1 & 1 & 0 \\
\hline $\begin{array}{l}73.87 \\
27\end{array}$ & $\begin{array}{l}\text { Typha domingensis Pers. } \\
\text { Urticaceae }\end{array}$ & $P$ & $\mathrm{He}$ & M,IS,ES & 0 & 0 & 1 & 0 & 0 \\
\hline 74.88 & Urtica urens L. & A & Th & Pan & 0 & 1 & 1 & 0 & 0 \\
\hline
\end{tabular}

$\mathrm{A}=$ annual, $\mathrm{B}=$ biennial, $\mathrm{P}=$ perennial herbs, $\mathrm{S}=$ shrub, $\mathrm{T}=$ tree, $\mathrm{Th}=$ Therophytes, $\mathrm{Hy}=$ Hydrophytes, $\mathrm{Hm}=\mathrm{Hemi}-$ cryptophytes, $\mathrm{Ph}=$ Phanerophytes, $\mathrm{Ch}=$ Chaemophytes, $\mathrm{Ge}=$ Geophytes, $\mathrm{He}=$ Helophyte, $\mathrm{Cosm}=$ Cosmopolitan, Pan = Pantropical, $\mathrm{Pal}=$ Palaeotropical, $\mathrm{M}=$ Mediterranean, IT = Irano-Turanian, SZ = Sudano-Zambezian, SS = SaharoSindian, ES $=$ Euro-Siberian, $\mathrm{S}=$ Sudanian, $1=$ presence, $0=$ absence 


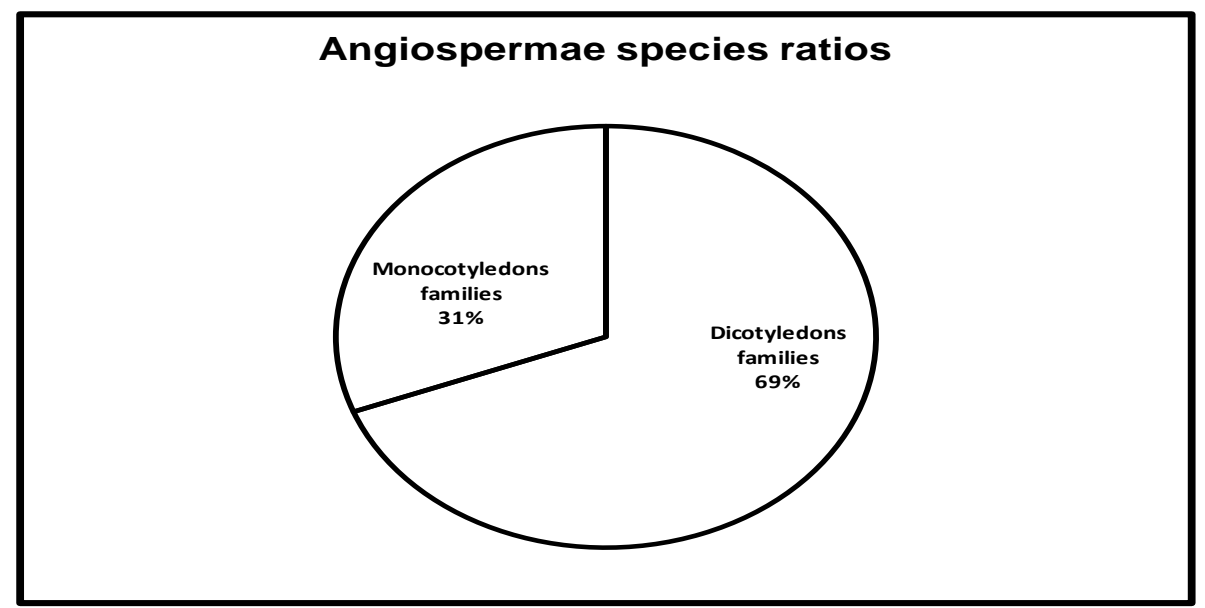

Fig. 2. Spectrum showed the total ratios of dicotyledons and monocotyledons families along Mariotteya Canal

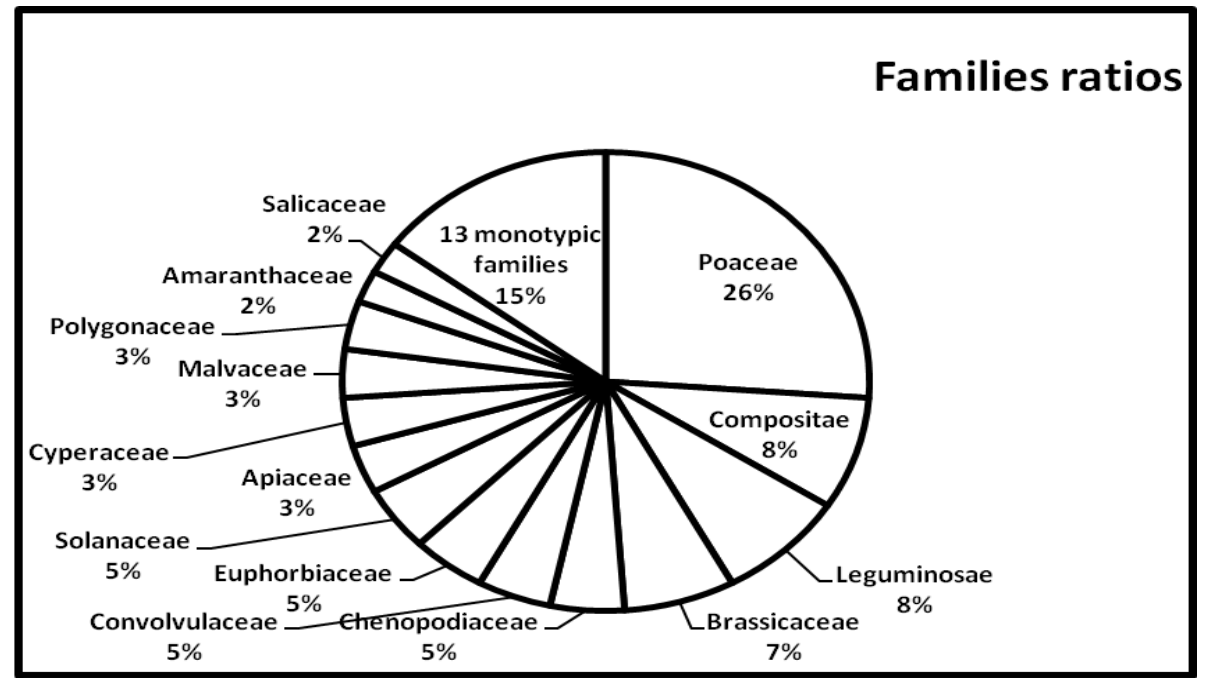

Fig. 3. Spectrum showed the families ratios of the recorded species along Mariotteya canal

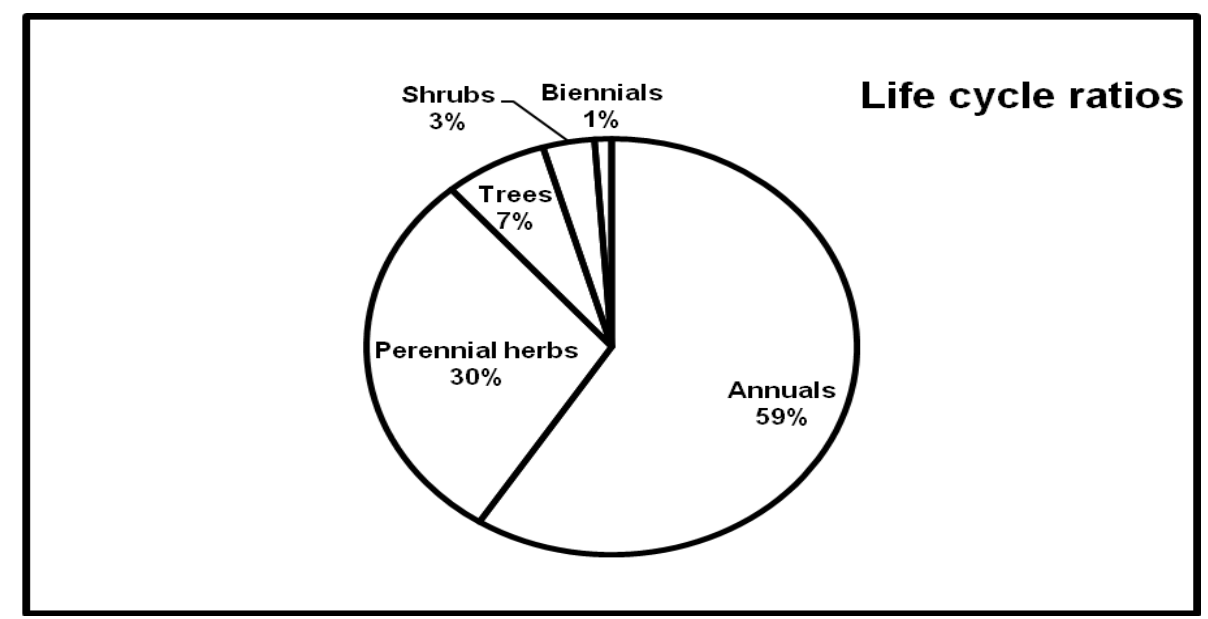

Fig. 4. Spectrum showed the life cycle ratios of the recorded species among the studied habitats along Mariotteya Canal 


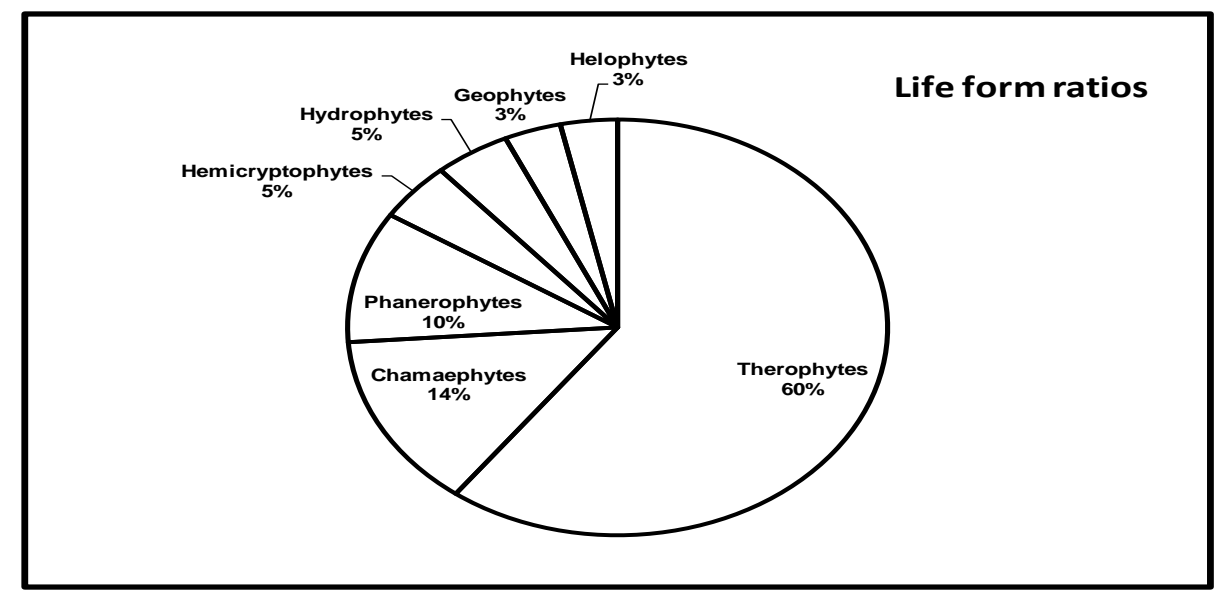

Fig. 5. Spectrum showed the life form ratios of the recorded species among the studied habitats along Mariotteya Canal

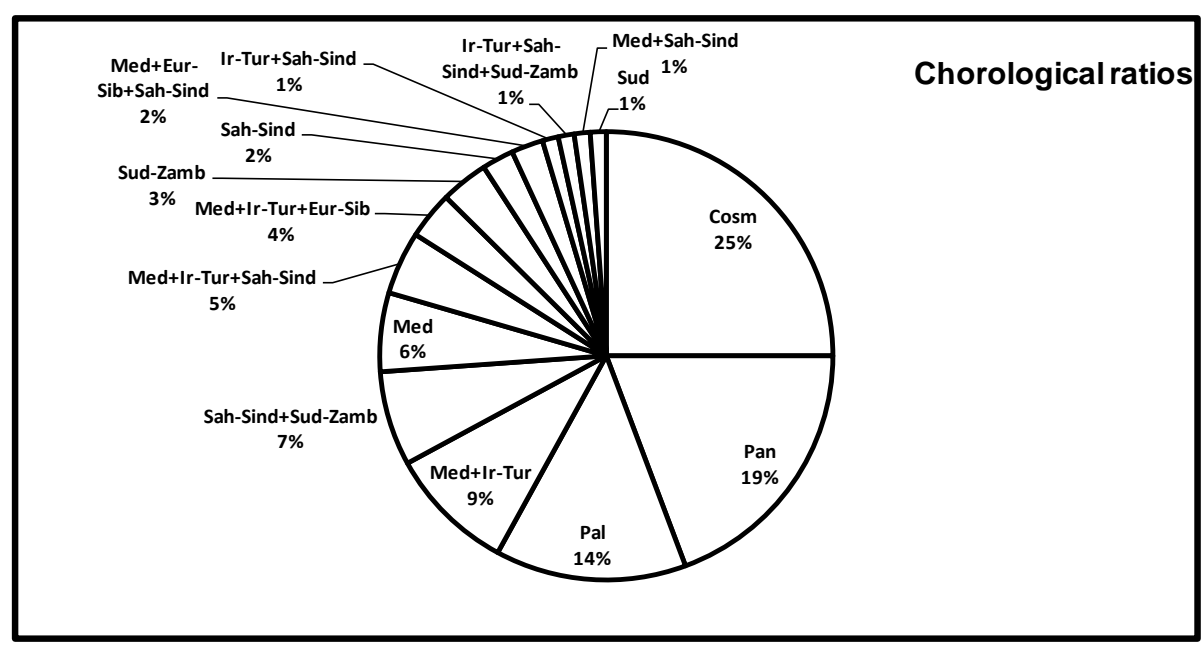

Fig. 6. Spectrum showed the chorological ratios of the recorded species among the studied habitats along Mariotteya Canal. Cosm= Cosmopolitan, Pan = Pantropical, Pal = Palaeotropical, Med = Mediterranean, Ir-Tur = Irano-Turanian, EurSib = Euro-Siberian, Sah-Sind = Sahro-Sindian, Sud-Zamb= SudanoZambezian, Sud = Sudanian

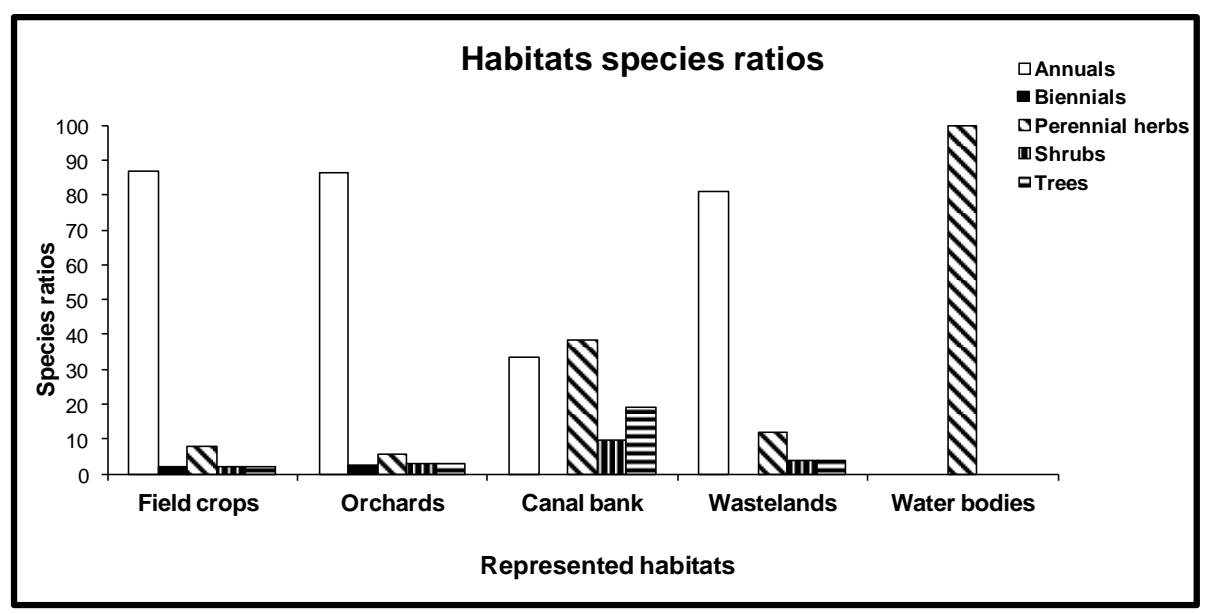

Fig. 7. Histogram showed the habitats species ratios of the recorded species among the studied habitats along Mariotteya Canal 
(1\%); respectively (Figure 6). On the whole, the total ratios of mono-regional, bi-regional, triregional and pluri-regional species were (12\%), (18\%), (12\%) and (58\%); respectively (Table 4).

\section{Habitats species ratios}

It was obvious that the annual species constituted the main bulk of the recorded species at the following habitats: field crops, orchards and wastelands along Mariotteya Canal. The weeds of field crops were represented by (86.54\%) followed by orchards $(86.49 \%)$, and wastelands $(80.77 \%)$. On the other hand; the perennial species constituted the main ratios at water bodies and canal banks habitats, where they represented by (100\%) and $(38.10 \%)$; respectively. The biennuals species were represented by $(2.70 \%)$ at orchards habitat and $(1.92 \%)$ at field crops habitat. Moreover, both of shrubs and trees were represented by (3.85\%) at wastelands, $(2.70 \%)$ at orchards and $(1.92 \%)$ at field crop habitats; while they did not record at water bodies' habitat (Figure 7).

\section{Major similrity among the studied habitats along Mariotteya Canal}

The data presented in Tables ( 2 \& 4) and dendrogram Figure (8), showed that, the recorded species among five represented habitats along Mariotteya Canal were separated into three groups (G1, G2 and G3). Group one included field crops and orchards habitats. Second group contained canal banks and wastelands habitats; while third group included water bodies' habitat. Moreover, field crops and orchards habitats showed the high- est degree of similarity ratio $(61.4 \%)$ followed by $(55.9 \%)$ between canal banks and wastelands habitats then (51.3\%) between orchards and canal banks habitats.

\section{Major dissimilarity among the studied habitats along Mariotteya canal}

The data presented in Table (3) and dendrogram Figure (8), showed that, there is no degree of similarity between the species of water bodies habitat and the other habitats namely; field crops, orchards, canal bank and wastelands habitats.

Table 2. Grouping of five studied habitats based on the numerical analysis of 88 species recorded along Mariotteya Canal

\begin{tabular}{|lc|}
\hline \multicolumn{1}{|c|}{ Studied habitats } & Groups \\
\hline Field crops and Orchards & G1 \\
Canal bank and Wastelands & G2 \\
Water bodies & G3 \\
\hline
\end{tabular}

Table 3. Proximity matrix showed the degree of species similarity distributed among the studied habitats along Mariotteya Canal

\begin{tabular}{|llllll|}
\hline \multirow{2}{*}{ Habitats } & \multicolumn{5}{c|}{ Proximity Matrix } \\
\cline { 2 - 6 } & $\begin{array}{l}\text { Field } \\
\text { crops }\end{array}$ & Orchards & $\begin{array}{c}\text { Canal } \\
\text { banks }\end{array}$ & Wastelands & $\begin{array}{l}\text { Water } \\
\text { bodies }\end{array}$ \\
\hline Field crops & 1.000 & & & & \\
Orchards & $\mathbf{0 . 6 1 4}$ & 1.000 & & & \\
Canal banks & 0.313 & $\mathbf{0 . 5 1 3}$ & 1.000 & & \\
Wastelands & 0.221 & 0.391 & $\mathbf{0 . 5 5 9}$ & 1.000 & \\
Water bodies & 0.000 & 0.000 & 0.000 & 0.000 & 1.000 \\
\hline
\end{tabular}

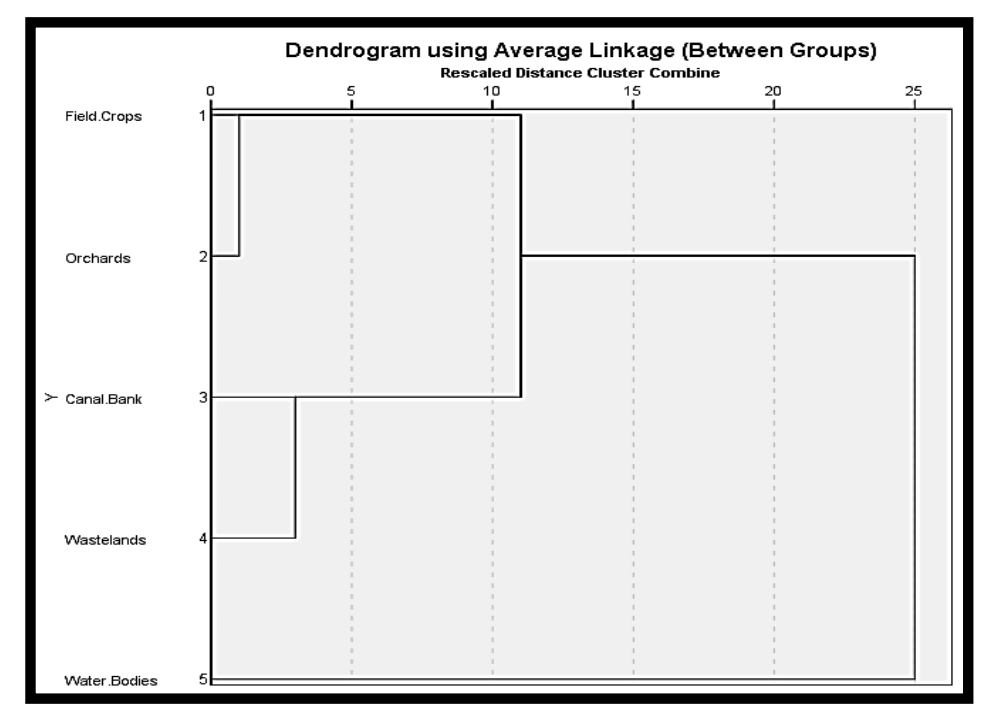

Fig. 8. Dendrogram showed the degree of similarity among the studied habitats along Mariotteya Canal 
Table 4. The chorological analysis of the recorded species along Mariotteya canal

\begin{tabular}{|lcc|}
\hline Chorotype & Species No. & Species ratio \\
\hline MONO-REGIONAL SPECIES & & \\
\hline Mediterranean & 5 & 6 \\
Sudano-Zambezian & 3 & 3 \\
Saharo-Sindian & 2 & 2 \\
Sudanian & 1 & 1 \\
Total of mono-regional species & $\mathbf{( 1 1 )}$ & $\mathbf{( 1 2 )}$ \\
BI-REGIONAL SPECIES & & \\
Mediterranean and Irano-Turanian & 8 & 9 \\
Saharo-Sindian and Sudano-Zambezian & 6 & 7 \\
Irano-Turanian and Saharo-Sindian & 1 & 1 \\
Mediterranean and Saharo-Sindian & 1 & 1 \\
Total of bi-regional species & $\mathbf{1 1 6}$ & $\mathbf{( 1 8 )}$ \\
TRI-REGIONAL SPECIES & & \\
Mediterranean, Irano-Turanian and Euro-Siberian & 4 & 5 \\
Mediterranean, Irano-Turanian and Euro-Siberian & 3 & 4 \\
Mediterranean, Euro-Siberian and Saharo-Sindian & 2 & 2 \\
Irano-Turanian, Saharo-Sindian and Sudano-Zambezian & 1 & 1 \\
Total of tri-regional species & $\mathbf{1 0}$ & $\mathbf{( 1 2 )}$ \\
PLURI-REGIONAL SPECIES & & \\
Cosmopolitan & 22 & 25 \\
Pantropical & 17 & 19 \\
Palaeotropical & 12 & 14 \\
Total of pluri-regional species & $\mathbf{( 5 1 )}$ & $\mathbf{( 5 8 )}$ \\
TOTAL & $\mathbf{8 8}$ & $\mathbf{1 0 0 \%}$ \\
\hline
\end{tabular}

\section{DISCUSSION}

\section{Floristic composition}

The recorded plant species represent about (4.13\%) of the Egyptian flora (Boulos 1995 and 2009). The three major distributed families were Poaceae, Compositae and Leguminosae. They comprised 37 taxa $(42.05 \%)$ of the total recorded species. These families were reported earlier by Mashaly et al (2009); Hamed et al (2012); Azer (2013) and Amer et al (2015) as the most frequent families in the studied areas. The families with the highest richness recorded by this study are compatible with the data of Quezel (1978) who reported that, Poaceae, Compositae, Leguminosae and Brassicaceae are among the most common families in the Mediterranean North African flora. Similar conclusion has been reached by Shaheen (2002) and Abd El-Ghani \& Fawzy (2006).

\section{Life cycle ratios}

Dominance of the perennial species and limited number of the annual ones along Mariotteya Canal seems to be a response to hot-dry climate, biotic and abiotic conditions that characterize the studied area. Heneidy \& Bidak (2001) and Abd El-Ghani et al (2013) reported the short life cycles of annual species probably lead to the frequent occurrence during the favorable seasons which supports the present conclusion. In accordance with this report, the composition of life cycle revealed that, perennials represent majority of recorded species in canal banks and water bodies habitats; while annuals species are the most common in field crops, orchards and wastelands. Abd El-Ghani and Fawzy (2006) explained these relationships based on the extensive root systems of the perennial species that are capable of utilizing water stored at differ- 
ent soil depths. Shaltout and Sharaf El-Din (1988) reported that, the flourishing of some species in different habitats is related to their great plasticity under different situations. These explanations are supported by the present investigation based on the studied species among different habitats. On the other hand, the low number of shrubs and trees, in the current study related to the high intensity of disturbance due to agricultural activities in the field crops and orchards; this fact also reported by Abd El-Ghani et al (2013) and (Amer et al 2015).

\section{Life form ratios}

The dominant life forms in the studied area were therophytes species $(60 \%)$ followed by Chamaephytes (14\%), then phanerophytes (10\%). As in the whole Egyptian flora, the therophytes were the most common life form (Hassib, 1951. Similar observation was cited by El-Ghareeb and Rezk (1989). Moreover, Heneidy and Bidak (2001) mentioned that the dominance of therophytes response to the hot dry climate and biotic influence.

\section{The chorological ratios}

The chorological ratios of the recorded taxa showed that cosmopolitan had the highest contribution (25\%), followed by Pantropical (19\%), then Palaeotropical (14\%). The current study confirmed by Zohary (1973) and Amer et al (2015). Moreover, the widely distributed species belonged to cosmopolitan, pantropical and palaeotropical chorotypes constituted (58\%) in the studied area. This indicated that the floristic structure of the study area was affected by human impact (Shaltout \& El-Fahar, 1991; Abd El-Ghani et al 2011 and Amer et al 2015). Also the current study was supported by (El-Hadidi, 1993) who concluded that the major percentage of the weed flora of Egypt is represented by cosmopolitan, pantropical and palaeotropical taxa. The presence of species related to different chorotypes categories was related to the position of Egypt at the border line between the Asiatic and African continents (Amer et al 2015). Also, El-Hadidi (1993) mentioned that the natural vegetation of Egypt belongs to SaharoSindian; Sudano-Zambezian; Mediterranean and Irano-Turanian regions.

\section{CONCLUSIONS}

In this study, the analysis of floristic composition concluded that, the recorded species were 88 species represents 74 genera and 27 families. The largest distributed families were Poaceae followed by Compositae and Leguminosae. Three species were recorded from each of Apiaceae, Cyperaceae, Malvaceae and Polygonaceae and two species from both Amaranthaceae and Salicaceae. In addition to, 13 families were monotypic. The dominant life cycle species were annuals followed by perennials species. Moreover, therophytes had the highest ratio of contribution followed by chamaephytes then phanerophytes. In addition to, the chorological ratios showed that cosmopolitan taxa had the highest contribution followed by pantropical then palaeotropical. The cluster analysis divided the studied habitats into three groups. Moreover, field crops and orchards habitats showed the highest degree of similarity. On the other hand, there was no similarity between species of water bodies' habitat and species of the other habitats. On the whole, the anthropogenic factors are operating together and reducing the chances of formation of new vegetation structure along Mariotteya Canal.

\section{REFERENCES}

Abd El-Ghani, M.M. and Fawzy, A.M. 2006. Plant diversity around springs and wells in five oases of the Western Desert, Egypt. International Journal of Agriculture and Biology 8, 249255.

Abd El-Ghani, M.M., Bornkamm, R., El-Sawaf, N. and Turkey, H. 2011. Plant species distribution and spatial habitat heterogeneity in the landscape of urbanizing desert ecosystem of Egypt. Urban Ecosystems 14. 585-616.

Abd El-Ghani, M.M.; Soliman, A. T.; Hamdy, R. and Bennoba, E. 2013. Weed flora in the reclaimed lands along the northern sector of the Nile Valley in Egypt. Turkish Journal of Botany 37, 464-488.

Ali, M.M., Hammad, A.H., Springuel, I.V. and Murphy, K.J. 1995. Environmental Factors Affecting Submerged Macrophyte Communities In Regulated Water Bodies In Egypt. Archiv Für Hydrobiologie, 133, 107-128.

Amer, W., Soliman, A. and Hassan, W. 2015. Floristic composition of Nile islands in Middle Egypt with special reference to the species migration route. Journal of American Science 11(6), 14-23. 
Angiosperm Phylogeny Group III 2009. An update of the Angiosperm Phylogeny Group classification for the orders and families of flowering plants: APG III". Botanical Journal of the Linnean Society $161(2), 105-121$.

Azer, S.A. 2013. Study on the plant diversity in Saqqara region, Giza, Egypt. Egyptian Journal of Biotechnology 45, 1-17.

Bliek, Van der A.M., El-Gharably, Z., Pieterse, A.H. and Scheepens, M.H. 1982. Observation of the phenology of Potamogeton pectinatus L. and other submerged weeds in irrigation systems in Egypt. Proc. EWRS 6th Symposium on Aquatic Weeds, EWRS, Wageningen, The Netherlands pp. 37-44.

Boulos, L. 1995. Flora of Egypt Checklist. Al Hadara Publishing, Cairo, Egypt, 10 p.

Boulos, L. 1999. Flora of Egypt. vol. 1 (Azollaceae - Oxalidaceae). Al-Hadara Publishing, Cairo, Egypt, 24p.

Boulos, L. 2000. Flora of Egypt. vol. 2 (Geraniaceae-Boraginaceae). Al-Hadara Publishing, Cairo, Egypt, 36 p.

Boulos, L. 2002. Flora of Egypt. vol. 3 (Verbinaceae - Compositae). Al-Hadara Publishing, Cairo, Egypt, 34 p.

Boulos, L. 2005. Flora of Egypt. vol. 4 (Monocotyledons: Alismataceae-Orchidaceae). Al Hadara Publishing, Cairo, Egypt, 102p.

Boulos, L. 2009. Flora of Egypt checklist (Revised annotated edition). Al Hadara Publishing Cairo Egypt, 16 p.

Chase, M.W. and Reveal, J.L. 2009. A phylogenetic classification of the land plants to accompany APG III". Botanical Journal of the Linnean Society, 161(2), 122-127.

El-Hadidi, M.N. 1993. Natural vegetation. In the Agriculture in Egypt, G.M. Craig (ed.), Oxford University Press, 39 p.

El Hadidi, M.N., Hosny, A.I. and El-Husseini, N. 1996. Some aspects of biodiversity of weed flora in the farm lands of Egypt in: The biodiversity of African plants. L.J.G. vander Maesen et al (eds), Kluwer Academic Publishers, Netherlands, 788-794.

El Hadidi, M.N. and Hosny, H.A. 2000. Flora Aegyptiaca. Vol. I, Part I, (M.N. El Hadidi ed.). Palm Press, Cairo, Egypt, 18 p.

El-Gharably, Z., Khattab, A.F. and Dubbers, F.A. 1982. Experience with grass carps for the control aquatic weeds in irrigation canals in Egypt. Proceeding $2^{\text {nd }}$ of International Symposium on Herbivorous Fish, 1982, EWRS, Wageningen, the Netherlands, pp. 17-26.
El-Ghareeb, R. and Rezk, R.M. 1989. A preliminary study on the vegetation of the coastal land at Bousseli (Egypt). Kuwait University (Science) 16, 115-127.

Feinbrun-Dothan, N. 1978. Flora Palaestina, (Part three). The Israel Academy of Science and Humanities,Israel Jerusalem Academic Press, Jerusalem, $90 \mathrm{p}$.

Feinbrun-Dothan, N. 1986. Flora Palaestina, (Part four). The Israel Academy of Science and Humanities, Israel Jerusalem Academic Press, Jerusalem, $123 \mathrm{p}$.

Hamed, S.T., Sheded, M.G. and Owis, M. 2012. Floristic composition of some riverian islands at Qena governorate- Egypt. Egyptian Journal of Botany 299- 322.

Hassib, M. 1951. Distribution of plant communities in Egypt. Bulletin of Faculty of Science, University of Fouad 1, Cairo, Egypt 29, 59-261

Haston, E., Richardson, J.E., Stevens, P.F., Chase, M.W. and Harris, D.J. 2009. The Linear Angiosperm Phylogeny Group (LAPG) III: a linear sequence of the families in APG III. Botanical Journal of the Linnean Society 161, 128131.

Heneidy, S.Z. and Bidak, L.M. 2001. Multipurpose plant species in Bisha, Asir region, Southwestern Saudi Arabia. King Saud University 13, 1126.

Khedr, A.A. and El-Demerdash, M.A. 1997. Distribution of aquatic plants in relation to environmental factors in the Nile Delta. Aquatic Botany, 56, 75-86.

Khedr, A.A. 1998. Vegetation zonation along Damietta estuary of the River Nile. Journal of Coastal Conservation, 4, 79-86.

Mashaly, I.A. and El-Ameir, Y.A., 2007. Hydrophytic Vegetation in the Irrigation and Drainage Canal System of the River Nile in Egypt. World Applied Sciences Journal 2(1), 49-61.

Mashaly, I.A., El-Habashy, I.E., El-Halawany, E.F., Omar, G. 2009. Habitat and plant communities in the Nile delta of Egypt 11. Irrigation and drainage canal bank habitat. Pakistan Journal of Biological Science 12, 885-895.

Mashaly, I.A., Khedr, A.A., Barakat, N. and Serag, M.S. 2003. On the ecology of water hyacinth community in the River Nile system of Egypt. Journal of Environmental Science, Mansoura Univ., 26, 229-249.

Mohamed, M.K. and Hassan, L.M. 1998. Studies on the plant life of the River Nile islands in Minia governorate. Proc. Sixth Eg. Bot. Conf., Giza, 3, 481-489. 
Quézel, P. 1978. Analysis of the flora of Mediterranean and Saharan Africa. Annales of the Missouri Botanical Garden 65, 479-534.

Raunkiaer, C. 1934. The life forms of plants and statistical plant geography. Clarendon Press, Oxford, $10 \mathrm{p}$.

Serag, M.S. and Khedr, A.A. 1996. The shoreline and aquatic vegetation of El Salam canal, Egypt. Journal of Environmental Science, Mansoura University, 11, 141-163.

Serag, M.S., Khedr, A.A., Zahran M.A. and Willis, A.J. 1999. Ecology of some aquatic plants in polluted water courses, Nile Delta, Egypt. Proc. $6^{\text {th }}$ International Conference. Journal of Arab Biologist Union, 9, 85-97.

Shaheen, A.M. 2002. Weed diversity of newly farmed land on the southern border of Egypt (Eastern and Eastern shores of Lake Nasser). Pakistan Journal of Biological Science 5, 602-608.

Shaheen, A.M., Shedaed, M.G., Hamed, A.I. and Hamada, F.A. 2004, Botanical diversity in the Flora of some islands in the Egyptian Nubia. Proceeding of First International Conference on Strategy of Egyptian Herbaria Marsh 9-11, Giza, Egypt, 162 p.

Shaltout, K.H. and El-Fahar, R. 1991. Diversity and phenology of weed communities in the Nile Delta region. Journal of Vegetation Science 2 , 385-390.

Shaltout, K.H. and Sharaf El-Din, A. 1988. Habitat types and plant communities along a transect in the Nile Delta region. Feddes Repertorium, 99, 153-162.
Shaltout, K.H., El-Kady H.F. and Al-Sodany, Y.M. 1995. Vegetation analysis of the Mediterranean region of the Nile Delta, Vegetatio, 166: 73-83.

Springuel, I.V. and Murphy, K.J. 1991. Euhydrophyte communities of the River Nile and its impoundments in Egyptian Nubia. Hydrobiologia, 218, 35-47.

SPSS, 2013. IBM SPSS Statistics for Windows, Version 22.0. Armonk, NY: IBM Corp., USA $25 \mathrm{p}$.

Täckholm, V. 1974. Students' Flora of Egypt. Second edition Published by Cairo University Printed by Cooperative Printing Company Beirut, 42 p.

Wickens, G.E. 1976. The flora of Jabal Marra (Sudan Republic) and its Geographical Affinities. Kew Bulletin, Additional Series V. London, HMSO, 13 p.

Zahran, M.A. and Willis, A.J. 2003. Plant life in the River Nile in Egypt. Mars Publishing House, Riyadh, Saudi Arabia, 20p.

Zohary, M. 1966. Flora Palaestina (Part one). The Israel Academy of Science and Humanities, Israel Jerusalem Academic Press, Jerusalem, $15 \mathrm{p}$.

Zohary, M. 1973. Geobotanical foundations of the Middle East. Vols. 1-2, Gustav Fischer Verlag, Stuttgar, 20 p.

Zohary, M. 1987. Flora Palaestina (Part two). The Israel Academy of Science and Humanities, Israel Jerusalem Academic Press, Jerusalem, $55 \mathrm{p}$. 